Acta bot. bras. (2):83-94 (1988) supl.

\title{
RESERVA BIOLÓGICA ESTADUAL DA PRAIA DO SUL (ILHA GRANDE, ESTADO DO RIO DE JANEIRO): LISTA PRELIMINAR DA FLORA
}

\author{
Dorothy Sue Dunn de Araujo (1) \\ Rogério Ribeiro de Oliveira (1)
}

\begin{abstract}
RESUMO - A Reserva Biológica Estadual da Praia do Sul está localizada na parte meridional da

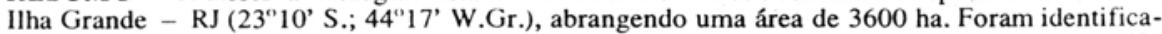
das nesta Reserva 5 comunidades vegetais, a saber: psamofila reptante de anteduna, mata de cordão arenoso, mata alagadiça de planície, manguezal e mata de encosta.

A fitofisionomia de cada comunidade está descrita, incluindo as espécies mais comuns e a área da Reserva ocupada por cada uma.

O levantamento florístico ainda incompleto constitui uma lista de cerca de 300 espécies: as 4 famílias mais importantes são: Leguminose, Rubiaceae, Orchidaceae, Bromeliaceae.
\end{abstract}

ABSTRACT - The Praia do Sul State Biological Reserve is located on Ilha Grande, State of Rio

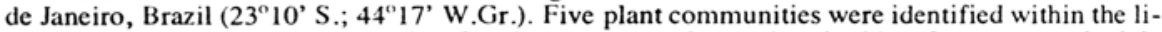
mits of this 3600 ha tract: creeping foredune psammophytes, beach ridge forest, coastal plain swamp forest, mangrove and montane forest.

The physiognomy of each community is described, including the most common species found and the area of occurrence within the Reserve.

A list of approximately 300 species has been compiled to date; the 4 most important families are: Leguminosae, Rubiaceae, Orchidaceae, Bromeliaceae.

Key-words: flora, plant communities, Biological Reserve.

\section{Introdução}

No litoral Sul do Estado do Rio de Janeiro a escarpa frontal da Serra do Mar atinge o oceano formando encostas abruptas e criando uma costa repleta de pequenas enseadas e planícies de áreas reduzidas. Um grande número de ilhas caracteriza este litoral, entre as quais a llha Grande, com uma superfície superior a $190 \mathrm{~km}^{2}$. Trata-se de um fragmento do maciço litorâneo isolado do continente por um canal de cerca de dois quilômetros de largura. O relevo é bastante acidentado e o Pico do Papagaio, com 959 m s.m. e a Serra do Retiro, com 1031 m s.m. constituem seus pontos de destaque (Maciel et al. 1984).

Na parte sudoeste da Ilha Grande está localizada a Reserva Biológica Estadual da Praia do Sul (RBEPS), abrangendo uma área de 3600 ha, coberta por uma vegetação que, na sua maior parte, está em bom estado de preservação. O clima da região é quente-úmido, sem estação seca (Fig. 1).

A RBEPS foi criada em dezembro de 1981 (Dec. Est. 4972), passando a ficar sob responsabilidade da Fundação Estadual e Engenharia do Meio Ambiente (FEEMA) em abril de 1982 (Dec. Est. 5444). Atualmente a World Wildlife Fund-US está financiando a construção da sede da Reserva.

(1) Fundação Estadual de Engenharia do Meio Ambiente (FEEMA). Departamento de Estudos e Projetos, C.P. 23.011, Rio de Janeiro, RJ - CEP 20.940 


\section{Materiais e Métodos}

A relação de espécies contida na presente contribuição é fruto de excursões periódicas realizadas na Reserva Biológica Estadual da Praia do Sul no período de 1979 a 1987, tendo maior intensidade de coleta entre 1983 e 1986.

Foram coletados ramos férteis das espécies botânicas, sendo prensados e desidratados segundo as técnicas usuais. As exsicatas foram depositadas no Herbário Alberto Castellanos (GUA), da Fundação Estadual de Engenharia do Meio Ambiente. A determinação do material foi feita através de consultas a chaves analíticas, por comparação ou por consultas a especialistas. Os nomes dos coletores encontram-se identificados por meio das seguintes siglas: DA: Dorothy Sue Dunn de Araujo; DP: Dorothea de Souza Pedrosa; HB: Helio Queiroz Boudet Fernandes; MC: Matilde Bucci Casari; RF: Ronaldo Fernandes de Oliveira e RR: Rogério Ribeiro de Oliveira.

As comunidades foram descritas baseadas em observações feitas no campo do hábito das plantas e após consulta ao mapa geológico preparado por Amador (1985).

\section{Resultados e Discussão}

\section{Descrição das Comunidades Vegetais}

A Ilha Grande, primitivamente, estava revestida de densa floresta tropical pluvial, desde os pontos culminantes até praticamente as áreas sob influência do mar. A paisagem foi bastante modificada pelos ciclos agro-econômicos que passaram pela ilha (café, canade-açúcar, legumes, frutos, grãos e gado) e, hoje em dia, a parte setentrional da ilha está coberta, principalmente, por vegetação degradada. Na vertente meridional, bem como no centro geográfico da llha, as matas estão em melhor estado de conservação (Magnanini $e t$ al. 1985).

As formações que ocorrem na planície da RBEPS constituem aproximadamente $22 \%$ da área total da Reserva. Devido à diversidade de condições geomorfológicas ali encontradas - praia, restinga (externa e interna), depósitos lagunares e aluviais, sedimentos de pântanos e manguezais (Amador 1985) - a composição florística varia muito de um local para o outro. Até o presente momento podem-se identificar, com segurança, 4 comunidades vegetais nesta planície: psamófila reptante da anteduna, mata de cordão arenoso, mata alagadiça de planície e manguezal. É muito provável que, com pesquisas mais detaIhadas, novas comunidades sejam delimitadas.

A comunidade de psamófilas reptantes ocorre na anteduna, localizada na parte superior da praia e é formada por uma faixa de largura variável, que pode atingir 10m ou ser temporariamente inexistente devido à erosão causada pelas fortes ressacas. Essa comunidade é constituída por espécies principalmente herbáceas, comuns a todo o litoral brasileiro, como, por exemplo Ipomoea pes-caprae, Alternanthera maritima, Panicum racemosum, etc., que crescem sobre a areia instável, formando um emaranhado relativamente denso de rizomas e estolões. Moitas isoladas de Scaevola plumieri, Chrysobalanus icaco, Cereus fernambucensis e Sophora tomentosa podem ser encontradas nos trechos da anteduna mais largos e antigos.

Afastando-se da praia, a vegetação apresenta outro aspecto, sendo a altura reduzida gradativamente conforme se aproxima a zona da anteduna. A ação do vento como modelador é visível e a vegetação constitui uma barreira praticamente impenetrável. Espécies características desta zona são Bromelia antiacantha, Polystichum adiantiforme, Ouratea cuspidata entre outras.

A mata de cordão arenoso, que cobre o cordão externo da restinga, é uma floresta baixa, de aproximadamente $10 \mathrm{~m}$ de altura, de troncos relativamente finos. As copas das 


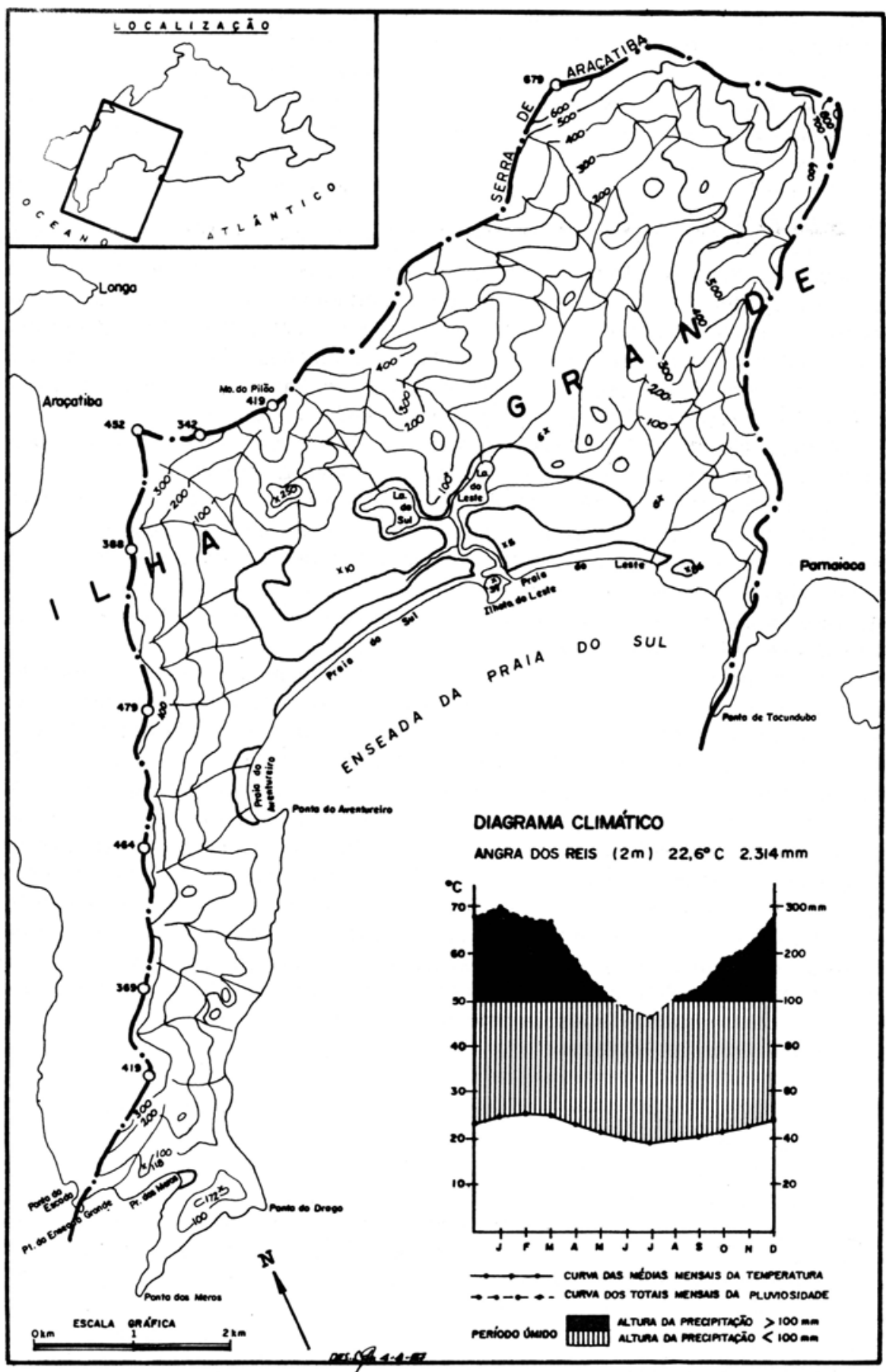

Fig. 1 - Localização da Reserva Biológica Estadual da Praia do Sul na llha Grande, RJ, delimitação da Reserva e diagrama climático referente a Angra dos Reis. 
árvores são cont'́guas na sua maioria, porém permitem a penetração de luz suficiente para o estabelecimento de um estrato inferior constituído por gravatás (e.g. Aechmea pectinata, Billbergia amoena, Nidularium microps forma acuminatum) e samambaias. As árvores mais comumente encontradas são: Rheedia brasiliensis, Tapirira guianensis, Ilex integerrima, Melanopsidium nigrum entre outras ainda não identificadas. No estrato arbustivo, encontrase Psychotria carthaginensis, P. barbiflora, Coccoloba glaziovii, Psidium cattleianum. A familia Araceae é muito bem representada nesta mata, tanto por espécies de hábito terrestre quando de hábito escandente.

Em alguns pontos, onde a vegetação do cordão arenoso já sofreu a ação do homem, a fisionomia é totalmente diferente, sendo caracterizada por moitas de arbustos com até 4$5 \mathrm{~m}$ de altura, intercaladas com espaços sem cobertura vegetal ou com arbustos e ervas esparsas. Algumas das espécies da mata também são muito comuns aqui, destacando-se entre elas Byrsonima sericea e Tapirira guianensis rebrotando de troncos grossos e cortados. Outras espécies arbustivas encontradas freqüentemente são Clusia cf. parviflora, Erythroxylum ovalifolium, Chaetocarpus pohlii e Gomidesia fenzliana.

A mata alagadiça de planície, de substrato úmido bem encharcado na época das chuvas, é uma formação bem mais robusta do que aquela descrita acima, sendo que as árvores podem atingir $20 \mathrm{~m}$ de altura. A presença de palmeiras e de grandes quantidades de epífitas nos galhos das árvores caracterizam esta mata, além de tapetes contínuos de gravatás no chão. As espécies até agora mais comumente encontradas são: Posoqueria latifolia, Tapirira guianensis, Guarea macrophylla subsp. tuberculata (estrato arbóreo); Psychotria brachyceras; P. hoffmannseggiana, Marlieria tomentosa (estrato arbustivo), Aechmea distichantha, Geophila repens (estrato inferior).

O manguezal é encontrado na baixada da Reserva, margeando os canais que ligam as lagoas do Sul e do Leste ao oceano. Forma uma faixa relativamente estreita no terreno onde a influência da maré se faz presente. As árvores atingem até $12 \mathrm{~m}$ de altura, sendo relativamente esparsa a sua distribuição. A composição específica é pouco diversificada como em todos os manguezais neotropicais, ocorrendo apenas três espécies arbóreas. Mais comumente encontram-se Rhizophora mangle e Laguncularia racemosa, sendo menos freqüente a Avicennia schaueriana. Em alguns trechos, os galhos destas árvores são carregados de epf́fitas (e.g. Aechmea nudicaulis var. nudicaulis, Anthurium scandens, Cattleya forbesii, Pleurothallis sp., Polystachya sp., Vriesea rodigasiana). Mais afastado das águas das lagoas ou dos canais, ocorre um ralo estrato herbáceo constituído por Fimbristylis spadicea, Cladium jamaicense e Triglochin sp.. Na transição do manguezal para terra firme é comum encontrar uma vegetação arbustiva composta de Hibiscus pernambucensis, Dalbergia ecastophylla e mais raramente Macherium sp., Miconia cf. hymenonervia e Myrcia multiflora var. glauscens.

Além destas 4 comunidades já identificadas para a planície da RBEPS, encontra-se nas elevações montanhosas que ocupam o restante da área (78\%), a mata de encosta. Vestígios como ruínas de antigas fazendas indicam que as encostas da RBEPS já foram palco de ocupação agrícola no passado, encontrando-se portanto em processo de regeneração. $O$ processo sucessional ocorrente se caracteriza por grande número de variantes em função de particularidades locais dos biótopos. Em alguns pontos encontram-se matas mais desenvolvidas, onde o estrato arbóreo atinge maiores proporções, com a presença de Erythroxylum sp., Cariniana sp. e Platymiscum sp.. As zonas de contato das formações orogênicas com a planície quaternária são caracterizadas pela dominância de Pothomorphe umbellata, no estrato inferior e Miconia hymenonervia, Schizolobium parahyba e Inga spp. no estrato superior.

Nas áreas onde ocorreu desmatamento mais severo, as espécies lenhosas pioneiras mais conspícuas são Miconia albicans, Peschiera sp. e Cybistax antisyphilitica. 
Lista preliminar da flora da Reserva Biológica Estadual da Praia do Sul

O levantamento florístico da RBEPS está ainda em estágio preliminar. Das comunidades apresentadas, a mata alagadiça de planície e a mata de encosta são as menos conhecidas botanicamente em função das dificuldades de acesso e de coleta.

Estas duas comunidades estão representadas com 31 e 104 espécies, respectivamente, enquanto as comunidades do cordão arenoso estão representadas com 144 espécies. A Tabela 1, que inclui somente as plantas vasculares, reune 302 espécies e variedades, representando 86 familias botânicas. As 4 familias contendo maior número de espécies são Leguminosae, Rubiaceae, Orchidaceae e Bromeliaceae (Tabela 2). Quando meIhor estudadas, as familias Myrtaceae e Araceae deverão assumir uma posição de maior importância, sendo então possível a comparação das espécies da presente lista com as de ecossıstemas similares em outras localidades geográficas.

\section{Referênclas Biblıográficas}

AMADOR, E. da S. 1985. Subsidios geologico/geomorfologicos à elaboração do Plano Diretor da Reserva Biologica Estadual da Praia do Sul. FEEMA. Rio de Janeiro.

MACIEL, N.C., ARAUJO, D.S.D. \& MAGNANINI, A. 1984. Reserva Biológica Estadual da Praia do Sul (Ilha Grande, Angra dos Reis, RJ): Contribuição para conhecimento da fauna e flora. Bolm FBCN 19: 126-148.

MAGNANINI, A., NEHAB, M.A.F. \& ARAUJO, D.S.D. 1985. Plano Diretor da Reserva Biologica Estadual da Praia do Sul - RJ. FEEMA. Rio de Janeiro.

Tabela 1 - LISTA PRELIMINAR DA FLORA VASCULAR DA RESERVA BIOLÓGICA ESTADUAL DO SUL, ILHA GRANDE, RJ

\section{ESPÉCIE}

FAMILIA

COLETOR, № COMUNIDADE

- Acicarpha spathulata $\mathrm{R}$. Br.

- Acrostichum sp.

- Adenocalyma comosum (Cham.) DC. var. lanceolatum Bur. \& K. Schum

- Aechmea distichantha Lem. var. distichantha

- Aechmea gracilis Lind.

- Aechmea nudicaulis (L) Griseb. var. nudicaulis

- Aechmea organensis Wawra

- Aechmea pectinata Baker

- Alchornea sp.

- Allophyllus sp.

- Alternanthera maritima (Mart.) St. - Hil.

- Amaioua intermedia Mart. ex Schult. \& Schult. var. brasiliana (Rich. ex DC.) Steyerm.

- Amaryllis spp.

- Anacardium occidentale L.

- Andira sp.

- Anthurium cf. pentaphyllum (Aubl.)

$$
\text { D. Don s.I. }
$$

- Anthurium scandens (Aubl.) Engl.

- Anthurium spp.

Aphelandra sp.
Calyceraceae

Polypodiaceae

DA 5822

$\mathrm{DA} s / \mathrm{n}^{\circ}$

Bignoniaceae RR 667

Bromeliaceae

Bromeliaceae

Bromeliaceae

Bromeliaceae

Bromeliaceae

Euphorbiaceae

Sapindaceae

Amaranthaceae

Rubiaceae

Amaryllidaceae

Anacardiaceae

Leg. Pap.

Araceae

Araceae

Araceae

Acanthaceae
DA 5873

DA 7310

DA 6475

DA 6176

MC 1132

DA 6221

DA 5720,6161

DA 4103,5881 psamófita reptante

manguezal

mata de encosta

mata alagadiça de planície

mata de cordāo aranoso

DA 6062, 4194 manguezal, restinga arbustiva, mata de cordăo arenoso mata de encosta mata de cordăo arenoso mata de encosta mata de encosta

psamófita reptante

mata de cordāo arenoso mata de cordão arenoso restinga arbustiva restinga arbustiva

mata de encosta manguezal mata de cordão arenoso, mata de encosta mata alagadiça de planície, mata de encosta 
- Arrabidaea leucopogon (Cham.) Sandw.

- Aspidosperma pyriopllum M. Arg.

- Asplenium sp.

- Asplundia sp.

- Astrocaryum aculeatissimum (Schott) Burr.

- Attalea dubia (Mart) Burr.

- Augusta longifolia (Spreng.) Rehder

- Avicennia schaueriana Stapf. \& Leech

- Baccharis sp.

- Bactris aff. escragnollei Glaz. ex Burr.

- Bathysa stipulata (Vell.) Presl.

- Begonia spp.

- Billbergia amoena (Lodd.) Lindley var. amoema

- Blutaparon portulacoides St. -Hil.

- Bomarea sp.

- Borreria cymosa C. \& S.

- Bromelia antiacantha Bertol.

- Byrsonima sericea DC.

- Cabralea canjerana (Vell.) Mart.

- Caesalpinia bonduc Roxb.

- Calathea sp.

- Calyptranthes lanceolata Berg.

- Canavalia paviflora Benth.

- Canavalia rosea (Sw.) DC.

- Canistrum lindenï (Reg.) Mez var. roseum (E. Morr.) L. B. Smith

- Capsicum sp.

- Capparis flexuosa (L) L.

- Casearia sp.

- Cassytha sp.

- Catasetum sp.

- Cattleya forbesii Lindl.

- Cattleya guttata Lindl.

- Cecropia sp.

- Cedrela sp.

- Celosia cymosa Seub.

- Centropogon sp.

- Centrosema sp.

- Cereus fernambucensis Lem.

- Cestrum sp.

- Chamaecrista rotundifolia Pers.

- Chioccoca nitida Benth.

- Chaetocarpus pohlii M. Arg.

- Chrysobalanus icaco L.

- Cissampelos sp.

- Cissus spp.

- Cladium jamaicense Crantz

- Clidemia hirta (L) Don

- Clidemia cf. parasitica Triana
Bignoniaceae

Apocynaceae

Polypodiaceae

Cyclanthaceae

Palmae

Palmae

Rubiaceae

Verbenaceae

Compositae

Palmae

Rubiaceae

Begoniaceae

Bromeliaceae

DA 5914

Amaranthaceae

Amaryllidaceae

Rubiaceae

Bromeliaceae

Malpighiaceae

Meliaceae

Leg. Caes.

Marantaceae

Myrtaceae

Leg. Pap.

Leg. Pap.

Bromeliaceae

Solanaceae

Capparaceae

Flacourtiaceae

Lauraceae

Orchidaceae

Orchidaceae

Orchidaceae

Moraceae

Meliaceae

Amaranthaceae

Campanulaceae

Leg. Pap.

Cactaceae

Solanaceae

Leg. Caes.

Rubiaceae

Euphorbiaceae

Chrysobala-

naceae

Menispermaceae DA 4181

Vitaceae

DA 6788,6472

Cyperaceae DA 5853

Melastomataceae DA 6056

Melastomataceae DA 6070 capoeira

restinga arbustiva

mata alagadiça de planície

mata de encosta

mata alagadiça de planície, mata de encosta

mata de enccsta

mata de encosta

manguezal

restinga arbustiva

mata de encosta

mata de encosta

mata de encosta, mata alagadiça de planície

mata de cordăo arenoso

psamofita reptante

mata de cordão arenoso

restinga arbustiva

restinga arbustiva, mata de cordão

arenoso

restinga arbustiva

mata de encosta

mata de cordão arenoso

mata de encosta

mata de encosta

mata de cordão arenoso

psamófita reptante

mata de cordão arenoso

mata de encosta

mata de cordão arenoso

mata alagadiça de planície

psamófita reptante

manguezal

mata de cordăo arenoso

restinga arbustiva, mata de

cordão arenoso

restinga arbustiva

mata de encosta

mata de encosta

mata alagadiça de planf́cie

restinga arbustiva

psamófita reptante

mata de encosta

restinga arbustiva

mata de cordão arenoso

restinga arbustiva

restinga arbustiva, psamófita reptante mata de encosta

restinga arbustiva, mata de encosta manguezal (transição) manguezal (transição) mata de encosta 
cont.

- Clidemia urc eolata DC.

- Clitoria sp.

- Clusia cf. parviflora (Sald.) Engl.

- Coccoloba glaziovii Lind.

- Codonanthe carnosa (Gard.) Hanst.

- Cordia magnoliifolia Cham.

- Costus sp.

- Coutarea hexandra (Jacq.) K. Schum.

- Cratylia sp.

- Croton compressus Lam.

- Cupania oblongifolia Mart.

- Cybistax antisyphillitica (Mart.) Mart. ex DC.

- Cyclopogon variegatus B. Rodr.

- Cyperus ligularis L. f.

- Cyrtopodium paranaense Schltr.

- Dahlstedtia pinnata (Benth.) Malme Leg. Pap.

- Dalbergia ecastophylla (L.) Taub. Leg. Pap.

- Dendropanax sp.

- Dichorisandra spp.

- Dioclea sp.

- Diodia radula (Willd. \& Hoffm. ex R. \& S.) C. \& S.

- Dioscorea sp.

- Dorstenia arifolia Lam.

- Dorypteris sp.

- Eleocharis sp.

- Emmeorrhiza umbellata (Spreng.) Schum.

- Endlicheria paniculata (Spr.) Macbr.

- Epidendrum spp.

- Epiphyllum phyllanthus (L) Haw.

- Erythrina sp.

- Erythroxylum ovalifolium Peyr.

- Eugenia brasiliensis Lam.

- Eugenia umbelliflora Berg.

- Euphorbia sp.

- Eurystylis sp.

- Euterpe edulis Mart.

- Faramea miltiflora A. Rich. ex DC. var. salicifolia (Presl.) Steyerm.

- Ficus insipida Willd.

- Ficus organensis (Miq.) Miq.

- Ficus trigona L. f.

Araliaceae

Melastom
Leg. Pap.

Guttiferae

Polygonaceae

Gesneriaceae

Boraginaceae

Zingiberaceae

Rubiaceae

Leg. Pap.

Euphorbiaceae

Sapindaceae

Bignoniaceae

Orchidaceae

Cyperaceae

Orchidaceae

Commelinaceae

Leg. Pap.

DA 6271

Rubiaceae

DA 4169

Dioscoreaceae

Moraceae

DA 4157

Polypodiaceae

DA 5882

DA 4187

Cyperaceae

Rubiaceae

Lauraceae

Orchidaceae

Cactaceae

Leg. Pap.

Erythroxylaceae

Myrtaceae

Myrtaceae

Euphorbiaceae

Orchidaceae

Palmae

DA 4134

DA 4201

DA 4183

DA 6499,6722

DA 4161

DA 6278

DA 6503,4122

DA 4125

DA 3091

DA 6311

DA 6804

HB 933

Rubiaceae

Moraceae

Moraceae

Moraceae

- Fimbristylis spadicea (L.) Vahl.

- Geonoma elegans Mart.

- Geophila repens (L.) I.M. Johnson

- Gomidesia fenzliana Berg.

- Gomidesia sp.

- Gomphrena vaga Mart.
Myrtaceae

Cyperaceae

Palmae

Rubiaceae

Myrtaceae

Amaranthaceae
DA 4186

DA 6126

DA 6487

DA 5930

DA 4143

DA 6193

DA 4108,4185

DA 6277,6743

RR 646

DA 6265 capoeira

capoeira

restinga arbustiva, mata de

cordão arenoso

restinga arbustiva

mata alagadiça de planície

mata de encosta

mata alagadiça de planície

\section{capoeira}

capoeira

mata de encosta

mata de encosta

mata de encosta

mata de cordăo arenoso

capoeira

restinga arbustiva

mata de encosta

restinga arbustiva, manguezal

mata de encosta

mata alagadiça de planície, mata de encosta

mata de encosta

restinga arbustiva

restinga arbustiva

mata de encosta

mata de encosta

manguezal (transição)

mata de encosta (orla)

mata de encosta

mata de cordão arenoso

restinga arbustiva

mata de çordăo arenoso

restinga arbustiva

restinga arbustiva

mata de cordão arenoso

psamófita reptante

mata de encosta

mata de encosta

mata de encosta

mata de encosta

mata de cordão arenoso

mata de cordão arenoso (orla)

manguezal

mata de encosta

mata alagadiça de planície

mata de encosta

restinga arbustiva, mata de

cordão arenoso

mata de encosta

capoeira 
cont.

- Gonioanthela axillaris (Vell.)

Font. et Schw.

- Guapira sp.

- Guarea macrophylla Vahl ssp. tuberculata (Vell.) Penn.

- Guatteria sp.

- Heisteria sp.

- Heliconia farinosa Raddi

- Heliconia spatho-circinnata Arist.

- Heliotropium sp.

- Hibiscus pernambucensis A. Camb. Malvaceae

- Hillia parasitica Jacq. ssp. nobilis (Vell.) Steyerm.

- Hubera ovalifolia DC.

- Humiria balsamifera Aubl.

- Hydrocotyle bonariensis Lam.

- Ichnanthus petiolatus (Nees) Doell Gramineae

- Ilex amara Bonpl.

- Ilex integerrima Reiss.

Aquifoliaceae

Aquifoliaceae

- Ilex paraguariensis St. -Hil.

Aquifoliaceae

- Inga edulis (Vell.) Mart. ex Benth. var. edulis

- Inga luschnathiana Benth.

- Inga sellowiana Benth.

- Ipomoea cairica (L) Sweet

- Ipomoea littoralis Boiss.

Rubiaceae

Melastomataceae

Humiriaceae

Umbelliferae

DA 5721

DA 5694

DA 6190

DA 4177

DA 4192

DA 6705

RR 629

DA 6779,7293

DA 5721

DA 5875

$\mathrm{DA} s / \mathrm{n}^{\circ}$

DA 5872

DA 5869

DA 3086,6477

DA 4195,6485

Leg. Mim.

DA 4179

Leg. Mim.

DA 4119

Leg. Mim.

DA 4145

Convolvulaceae

Convolvulaceae

DA 4203

- Ipomoea pes-caprae (Lᄂ) Sweet

Convolvulaceae

- Ipomoea phyllornega (Vell.) House Convolvulaceae

$D A s / n$

$D A s / n^{\circ}$

DA 5856,

RR 635

Bignoniaceae

Asclepiadaceae

DA 5844

Convolvulaceae

DA 6094, 6114

RR 471

Combretaceae DA s $/ n^{2}$

Verbenaceae

DA 5823

Melastomataceae DA 5893

Melastomataceae DA 4174

Orchidaceae

DA 5862,6782

- Liparis elata Lindl.

- Lipostoma capitatum (R. Grah.) D. Don.

- Lophophytum mirabile Schott \& Endl.

- Lundia cordata DC.

- Lycopodium sp.

- Machaerium sp.

- Malaxis sp.

- Mandevilla funiformis (Vell.) k. Schum.

Rubiaceae

DA 5842

Balanophoraceae RR 672

Bignoniaceae

DA 6215

Lycopodiaceae

DA 6754

Leg. Pap.

DA 6216

Orchidaceae

DA 6187

Apocynaceae

DA 5863

- Manilkara subsericea (Mart.) Dub.

- Maranta sp.
Sapotaceae

DA 4126

Marantaceae mata de cordăo arenoso (orla) restinga arbustiva

mata alagadiça de planície, mata de encosta

mata de encosta, mata de cordão arenoso

restinga arbustiva

mata de encosta

mata de encosta

capoeira

manguezal

mata de cordăo arenoso

capoeira

mata de cordão arenoso

psamofita reptante

mata de cordăo arenoso

restinga arbustiva

restinga arbustiva, mata de

cordão arenoso

restinga arbustiva, mata de

cordăo arenoso

mata de encosta

restinga arbustiva

restinga arbustiva

capoeira

psamófita reptante

psamófita reptante

mata de cordăo arenoso

(orla)

mata de cordăo arenoso

restinga arbustiva, mata de

encosta (orla)

capoeira

manguezal

mata de cordāo arenoso (orla)

mata de encosta

mata de encosta

mata de cordăo arenoso,

mata de encosta

mața alagadiça de planície

mata de encosta

mata de cordão arenoso

restinga arbustiva

manguezal (transição)

restinga arbustiva

restinga arbustiva

restinga arbustiva

mata de encosta 
- Mariscus pedunculatus (R. Br.)

$$
\text { T. Koyama }
$$

- Marlieria tomentosa Camb.

- Marsdenia sp.

- Masdevallia sp.

- Matayba sp.

- Maxillaria aff. discolor Reichb.

- Maytenus sp.

- Melanopsidium nigrum Cels.

- Merremia dissecta (Jacq.) Hallier

- Merremia macrocalyx (R. \& P.) O'Donell

- Miconia cf. albicans (Sw.) Triana

- Miconia calvescens DC.

Cyperaceae DA $s / n^{2}$

Myrtaceae DA 6304

Asclepiadaceae DA6734

Orchidaceae DA 5713

Sapindaceae DA 6496

Orchidaceae DA 6501

Celastraceae DA 7308

Rubiaceae DA 4123

Convolvulaceae DA 5847

Convolvulaceae DA 3070

Melastomataceae DA 6112

Melastomataceae DA 6269

- Miconia dodecandra (Desr.) Cogn. Melastomataceae DA 6162

- Miconia cf. hymenonervia (Raddi) Cogn.

- Miconia prasina (Sw.) DC.

- Mimosa sp.

- Mollinedia longifolia Tul.

- Mollinedia schottiana (Spr.) Perk.

- Monstera sp.

- Mucuna sp.

- Myrcia multiflora (Lam.) DC.

- Myrcia multiflora (Lam.) DC. var glauscens (Berg) Legr.

- Myrcia racemosa (Berg) Kiaersk.

- Myrcia racemosa (Berg) Kiaersk. var. gaudichaudiana (Berg) Legr.

- Myrcia recurvata Berg

- Nematanthus fissus (Vell.) L. Skog

- Neoregelia cruenta (R. Grah.) L.B. Smith

- Nidularium innocentï Lem. var. paxianum (Mez) L.B. Smith

- Nidularium microps E. Morr. ex Mez forma acuminatum Per. et Lem.

\section{- Norantea brasiliensis Choisy}

Melastomataceae DA 6067

Melastomataceae DA 6166

Leg. Mim.

DA 6222

Monimiaceae

DA 6071

Monimiaceae

DA 6204

Araceae

Leg. Pap.

Myrtaceae

DA 7313

DA 5888, 4109

Myrtaceae

DA 6476

Myrtaceae

DA 6063

DA 6107

Myrtaceae

DA 6299

Myrtaceae

DA 6495

Gesneriaceae

DA 4209

Bromeliaceae

DA 5702

Bromeliaceae

DA 6108

Bromeliaceae

DA 6124,6725

Marcgraviaceae

DA 4202

- Octomeria aff. grandiflora Lindl.

Orchidaceae

DA 6502

- Oeceoclades maculata (Lindl.) LindI Orchidaceae

DA 6127

- Oncidium ciliatum Lindi.

Orchidaceae

DA 6801

- Opuntia vulgaris Mill.

Cactaceae

DA 4168

- Ormosia arborea (Vell.) Harms

Leg. Pap.

DA 6103

- Ossaea marginata (Desr.) Triana

Melastomataceae DA 5895

- Ottonia anisum Spr.

- Ottonia macrophylla Kunth.

Piperaceae

DA 6125

Piperaceae

DA 5727

- Ouratea cuspidata (St. -Hil.) Engl.

- Oxalis fruticosa Kunth

Ochnaceae

DA 6474

Oxalidaceae

DA 6717

- Oxypetalum alpinum (Vell.) Font. \& Schw. var. alpinum

Asclepiadaceae

DA 6699

- Oxypetalum banksï R. \& S. ssp. banksii

Asclepiadaceae

DA 5699 psamófita reptante

mata alagadiça de planície

mata de cordão arenoso

mata de cordão arenoso

mata de cordão arenoso

mata de cordão arenoso

restinga arbustiva

mata de cordăo arenoso

mata de cordão arenoso (orla)

capoeira

mata de encosta

mata de encosta

mata de encosta

manguezal (transiçăo)

mata de encosta

mata de cordão arenoso

mata de encosta

mata de encosta

mata de encosta

restinga arbustiva, mata de

cordão arenoso

restinga arbustiva

manguezal

mata alagadiça de planície

mata alagadiça de planície mata de cordão arenoso.

mata alagadiça de planície

mata de cordão arenoso

mata alagadiça de planície

mata de encosta, mata de cordăo arenoso

restinga arbustiva

mata de cordão arenoso

mata de escosta

mata de cordão arenoso

mata de cordão arenoso

mata de cordão arenoso

mata de escosta

mata de encosta

mata de encosta

restinga arbustiva

mata de encosta

mata alagadiça de planície (orla)

mata de cordão arenoso 
cont.

- Paspalum vaginatum Sw.

- Passiflora edulis Sims.

- Passiflora mucronata Lam.

- Passiflora organensis Gardn.

- Paullinia coriacea Casar.

- Pavonia schiedeana Steudel

- Peperomia glabella (Sw.) A. Dietr. Piperaceae

- Peperomia urocarpa Fisch. \& Mey. Piperaceae

- Pera sp.

- Pereskia aculeata Miller

- Pharus sp.

- Phaseolus sp.

- Philodendron sp.

- Phoradendron sp.

- Pilocarpus sp.

- Piper amplum Kunth

- Piper rivinoides Kunth

- Piper solmsianum C. DC.

- Piptocarpha sp.

- Pisonia sp.

- Pitcairnia flammea Lindl.

- Pithecellobium sp.

- Pleurothallis spp.

- Polygala cyparissias St. - Hil. \& Moq. Polygalaceae

- Polygala laureola St.-Hil. \& Moq.

- Polygala paniculata KI. ex Benn

- Polypodium triseriale Sw.

- Polystachya sp.

- Polystichum adiantiforme (Forst.) J. Sm.

- Portea petropolitana (Wawra)

Mez var. petropolitana

- Posoqueria cf. latifolia (Rudge) R. \& S.

- Pothomorphe umbellata (L.) Miq.

- Prescottia sp.

- Psidium cf. cattleianum Sabine

- Psychotria barbiflora DC.

Psychotria brachyceras M. Arg. Psychotria carthaginensis Jacq.

- Psychotria deflexa DC.

- Psychotria hoffmannseggiana (Willd. e R. \& S.) M. Arg.

- Psychotria leiocarpa Cham .\& Schlecht.

- Psychotria nuda (Cham. et Schlecht.) Wawra

- Psychotria racemosa (Aubl.) Raeusch.

- Psychotria stachyoides Benth.

- Quararibea sp.

- Quesnelia quesneliana (Brongn.) L.B. Smith
Gramineae

Passifloraceae

Passifloraceae

Passifloraceae

Sapindaceae

Euphorbiaceae

Cactaceae

Gramineae

Leg. Pap.

Araceae

Loranthaceae

Rutaceae

Piperaceae

Piperaceae

Piperaceae

Compositae

Nyctaginaceae

Bromeliaceae

Leg. Mim.

Orchidaceae

Polygalaceae

Polygalaceae

Polypodiaceae

Orchidaceae

Polypodiaceae

Bromeliaceae

Rubiaceae

Piperaceae

Orchidaceae

Myrtaceae

Rubiaceae

Rubiaceae

Rubiaceae

Rubiaceae

Rubiaceae

Rubiaceae

Rubiaceae

Rubiaceae

Rubiaceae

Bombacaceae

Bromeliaceae
DA 4211

DA 6483

DA 6285

DA 7279

DA 6307,6180

DA 6088

DA 4120,5711

DA 5884

DA 6101

DA 6183

DA 6090

DA 4117

DA 5726

DA 4141

DA 4193

DA 4178

DA 5894

DA 5901

DA 5705

DA 4139

DA 4205

DA 6790

DA 6061,6802

DA 4166

DA 4182

DA 6053

DA 6786

DA 6060,6102

DA 4164

DA 6492

DA 6296

DA 7324

DA 6966

DA 4198

DA 6076,6177

DA 4140

DA 5707,6170

DA 6172

DA 6479

DA 6077

RR 731

DA 3080

DA 6224

DA 6207

DA 6692 psamofita reptante

restinga arbustiva

restinga arbustiva

restinga arbustiva

mata de cordão arenoso, mata de encosta (orla)

mata de encosta

restinga arbustiva, mata de

cordão arenoso

mata de encosta

mata de cordão arenoso

restinga arbustiva

mata de encosta

restinga arbustiva

mata de encosta (orla)

mata alagadiça de planície mata de cordão arenoso (orla)

mata de encosta

mata de encosta

mata de encosta

mata alagadiça de planície

mata alagadiça de planície

mata de encosta (orla)

restinga arbustiva

manguezal; mata de cordão

arenoso

psamófita reptante

mata de encosta

manguezal (transiçăo)

restinga arbustiva

manguezal; mata alagadiça

de planície

mata de cordão arenoso (orla)

mata alagadiça de planície

mata de cordão arenoso

mata de encosta

mata de encosta

mata de cordão arenoso, restinga arbustiva.

mata de cordão arenoso, mata de encosta mata alagadiça de planície

mata alagadiça de planície, mata de encosta mata de encosta

mata alagadiça de planície

mata de encosta

mata de encosta

mata de encosta

mata de cordão arenoso

mata de encosta

mata de cordão arenoso 
- Randia armata (Sw.) DC.

- Rapanea sp.

- Rheedia brasiliensis PI. \& Tr.

- Rhipsalis capilliformis Web.

- Rhipsalis elliptica G.A. Lindberg

- Rhipsalis grandiflora Haw

- Rhipsalis oblonga Loefgr.

- Rhipsalis platycarpa (Zucc.) Pfeiff.

- Rhipsalis rhombea Pfeiff.

- Rhizophora mangle L.

- Rudgea cf. microcephala M. Arg.

- Ruppia maritima L. sensu lato

- Scaevola plumieri (L.) Vahl

- Scaphyglottis sp.

- Schinus terebinthifolius Raddi

- Schizolobium parahyba (Vell.) Blake

- Selenicereus sp.

- Serjania caracasana (Willd.) Radlk.

- Serjania cuspidata Camb.

- Serjania dentata (Vell.) Radlk.

- Siparuna apiosyce (Mart.) A. DC.

- Sloanea garckeana Schum.

- Smilax brasiliensis Spreng.

- Smilax quinquenervia Vell.

- Smilax rufescens Griseb.

- Solandra viridiflora Sims.

- Solanum argenteum Dun.

- Sophora tomentosa L.

- Spigelia sp.

- Sporobolus virginicıs Kunth

- Stelis sp.

- Stenotaphrum secundatum (Walt.) Kuntze

- Stigmaphyllon sp.

- Swartzia sp.

- Syagrus romanzoffiana (Cham.) Glassman

- Tabebuia sp.

- Talinum sp.

- Tapirira guianensis Aubl.

- Temnadenia stellaris (Lindl) Miers. Apocynaceae

- Tetrapterys phlomoides (Spr.) Nied. Malpighiaceae

Goodeniaceae

Orchidaceae

Anacardiaceae

Leg. Caes.

Cactaceae

Sapindaceae

Sapindaceae

Sapindaceae

Monimiaceae

Ebenaceae

Smilacaceae

Smilacaceae

Smilacaceae

Solanaceae

Solanaceae

Leg. Pap.

Loganiaceae

Gramineae

Orchidaceae

Gramineae

Malpighiaceae

Leg. Caes.

Palmae

Bignoniaceae

Portulacaceae

DA 6473

DA 4155

DA 6703

DA 3077

- Tibouchina estrellensis (Raddi) Melastomataceae DA 6119 Cogn.

- Tibouchina cf. viminea (Don) Cogn. Melastomataceae DA 6091

- Tillandsia geminiflora Brongn.

- Tillandsia tenuiflora L. var. surinamensis (Mez) L. B. Smith.

- Tocoyena bullata (Vell.) Mart.

- Tournefortia gardneri A. DC.

- Trema micrantha (L.) Blume

- Trimezia sp.

- Triglochin sp.

- Typha domingensis Pers.
Bromeliaceae DA 6210

Bromeliaceae

DA 6762

Rubiaceae

DA 4167

Boraginaceae

DA 4163

Ulmaceae

DA $s / n^{\circ}$

Iridaceae DA 4206

Sheuchzeriaceae DA 3092

Typhaceae DA s/n? mata de encosta (orla)

mata de cordão arenoso

mata de cordão arenoso

mata de cordão arenoso

mata de encosta

manguezal

restinga arbustiva

mata de cordão arenoso

mata de cordão arenoso

manguezal

mata de encosta

manguezal

psamofita reptante

mata de encosta

mata de cordăo arenoso (orla)

mata de encosta

mata de cordăo arenoso

restinga arbustiva

restinga arbustiva

mata de encosta

mata de encosta

mata de encosta

mata alagadiça de planície

mata de encosta

restinga arbustiva

mata de encosta

capoeira

psamofita reptante

mata alagadiça de planície

psamófita reptante

mata alagadiça de planície

psamofita reptante

restinga arbustiva

mata de encosta

mata de encosta

mata de cordão arenoso

mata de encosta

restinga arbustiva

mata de cordāo arenoso (orla)

mata de cordăo arenoso,

mata de encosta

mata de encosta

capoeira

mata de cordão arenoso

mata de cordão arenoso

mata de cordão arenoso (orla)

restinga arbustiva

restinga arbustiva,

mata de encosta

mata de cordão arenoso

manguezal

mata alagadiça de planície (orla) 


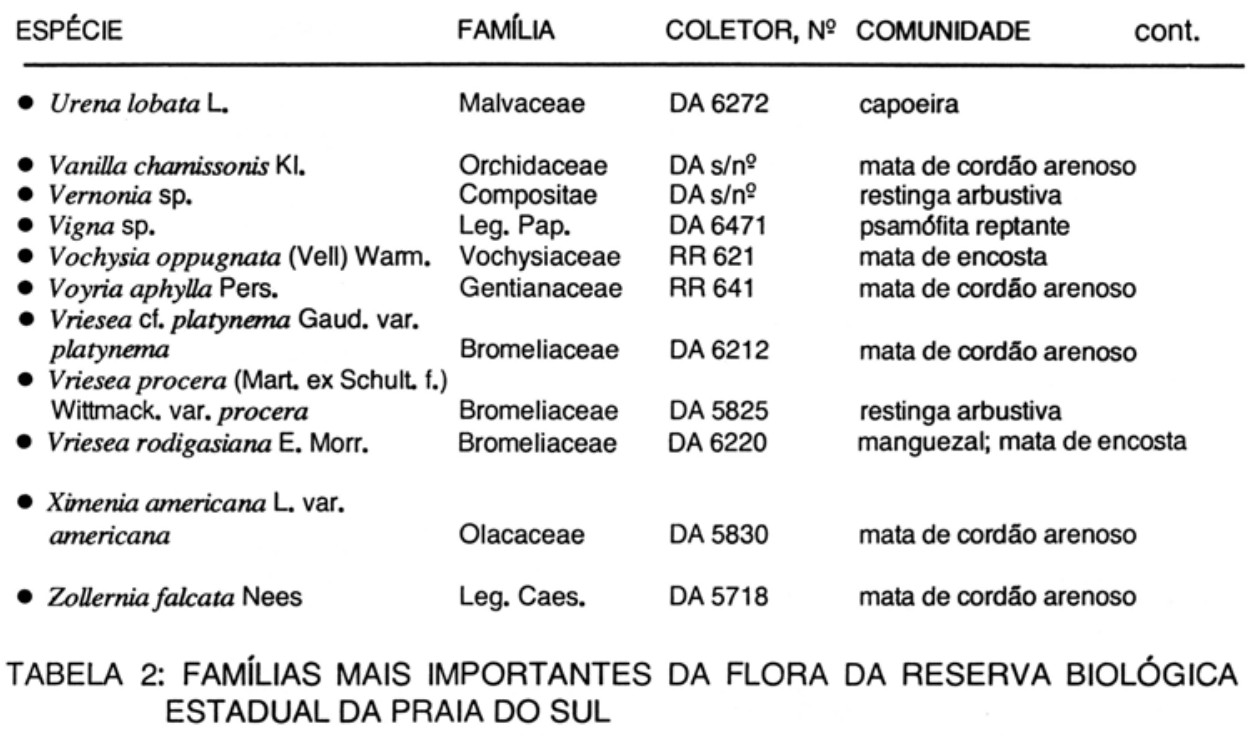

FAMILIA

TOTAL DE ESPÉCIES
PORCENTAGEM DO TOTAL

Leg. Pap.

Caes.

Mim.

Rubiaceae

Orchidaceae

Bromeliaceae

Melastomataceae

Cactaceae

Myrtaceae

Piperaceae

Convolvulaceae

Sapindaceae

Bignoniaceae

Gramineae

\section{Palmae}

Asclepiadaceae

Araceae

Cyperaceae

Euphorbiaceae

Polypodiaceae

Moraceae

2 Familias com 4 spp. 